\title{
Article \\ Changes to Physical Activity, Sitting Time, Eating Behaviours and Barriers to Exercise during the First COVID-19 'Lockdown' in an English Cohort
}

\author{
Lindsy Kass *, Terun Desai ${ }^{+}\left(\mathbb{D}\right.$, Keith Sullivan, Daniel Muniz and Amy Wells ${ }^{\dagger}$ \\ Life and Medical Science, University of Hertfordshire, Hertfordshire AL10 9EU, UK; t.desai@herts.ac.uk (T.D.); \\ k.sullivan3@herts.ac.uk (K.S.); d.muniz@herts.ac.uk (D.M.); a.v.wells@herts.ac.uk (A.W.) \\ * Correspondence: 1.s.kass@herts.ac.uk \\ † These authors corresponded equally to this work.
}

check for updates

Citation: Kass, L.; Desai, T.; Sullivan, K.; Muniz, D.; Wells, A. Changes to Physical Activity, Sitting Time, Eating Behaviours and Barriers to Exercise during the First COVID-19 'Lockdown' in an English Cohort. Int. J. Environ. Res. Public Health 2021, 18, 10025. https://doi.org/10.3390/ ijerph181910025

Academic Editors:

Maria Yavropoulou, José

Carmelo Adsuar Sala and Dimitros Paraskevis

Received: 8 July 2021

Accepted: 4 September 2021

Published: 24 September 2021

Publisher's Note: MDPI stays neutral with regard to jurisdictional claims in published maps and institutional affiliations.

Copyright: (c) 2021 by the authors. Licensee MDPI, Basel, Switzerland. This article is an open access article distributed under the terms and conditions of the Creative Commons Attribution (CC BY) license (https:// creativecommons.org/licenses/by/ $4.0 /)$.
Abstract: This study aimed to determine the effect of the first English national COVID-19 lockdown on physical activity (PA), sitting time, eating behaviours and body mass in an adult cohort. This was further examined to determine whether conforming to recommended guidelines on PA and sedentary behaviour was improved. Based on an online survey $(n=818)$ incorporating the International Physical Activity Questionnaire Short Form (IPAQ-SF), self-reported body mass change showed that in 32.2\% of participants body mass increased, with $39.1 \%$ reporting an increase in food intake. Never exercising at the gym or undertaking an exercise class (online or live), increased by $50.8 \%$ during lockdown, with $53.5 \%$ changing from exercising frequently to never exercising, suggesting a lack of engagement with online and home workouts. However, outdoor running and cycling $>2$ times/week increased by $38 \%$ during lockdown. Walking at least 30 min continuously on $>2$ occasions/week increased by $70 \%$ during lockdown with minimum 10-min walks on 7 days per week increasing by $23 \%$. The lockdown had a negative impact on sitting time ( $>8 \mathrm{~h}$ a day), which increased by $43.6 \%$ on weekdays and $121 \%$ at weekends. Furthermore, sitting $<4 \mathrm{~h}$ /day decreased during lockdown $(46.5 \%$ and $25.6 \%$ for weekdays and weekends, respectively). Those citing tiredness or lack of time as a barrier to exercise reduced by $16 \%$ and $60 \%$, respectively, from pre-lockdown to during lockdown. More of the sedentary group met the Public Health England PA recommendations, however most participants still did not meet the UK Government guidelines for PA. Improvements in health per additional minutes of physical activity will be proportionately greater in those previously doing $<30 \mathrm{~min} /$ week, the area where most improvements were found although, conversely sitting time was greatly increased. This study may assist in informing whether future lifestyle changes could improve the health of the population.

Keywords: COVID-19; behaviours change; physical activity; barriers to exercise; sitting time

\section{Introduction}

The World Health Organization (WHO) declared a Public Health Emergency of International Concern due to the outbreak caused by severe acute respiratory syndrome coronavirus 2 (SARS-CoV-2) on 30 January 2020, and a pandemic on 11 March 2020 [1]. To reduce the speed of the transmission of the SARS-CoV-2, several governments implemented measures to limit the movements of its citizens. The United Kingdom (UK) Government enforced a lockdown on the UK population from 23 March 2020, resulting in most adults and children staying at home, only allowed to leave the household for certain activities [2] These measures were relaxed in mid-May 2020 and enforced again in November 2020.

During the first phase of lockdown (23 March to 13 May 2020) many people were furloughed or allowed to work from home, resulting in a change in living conditions, and lifestyle. The lockdown itself posed challenges for those working with school or nursery age children, as childcare was not available, except for children of key workers. 
Consequently, this may have affected the time devoted to family life and engagement in physical activity (PA). As the lockdown impacted people's working patterns and amount of time spent at home, it potentially allowed for greater time to undertake physical activity. However, gyms and public sport centres were closed, so all exercise became self-motivated without the normal physical and social support of a structured training environment. This was the first time since the Second World War that a mass change in lifestyle had been enforced on the UK population and curfew measures have been shown to affect several aspects of lifestyle [3].

The UK Government recommends that adults aged 19-64 years should undertake 150 min of moderate physical activity or 75 min of vigorous physical activity per week [4]. Walking guidelines by Public Health England [5] recommend $30 \mathrm{~min}$ a day moderate intensity physical activity, to include walking, 5 days a week (or 150 min over a week) or 10 min of brisk walking per day for 7 days per week).

Physical inactivity has been defined as less than $30 \mathrm{~min}$ of physical activity a week [5]. Sedentary behaviour as defined by the World Health Organisation (2020) refers to behaviours in which energy expenditure is very low and sitting or lying is the dominant mode of posture [6]. Both physical inactivity and sedentary behaviour contribute to cardiovascular disease [7] as well as increasing the risk of obesity, type 2 diabetes and stroke with inactivity contributing to one in six deaths in the UK, the same number as smoking [8].

However, the Health Survey for England [9] showed that in 2016, only 66\% of men and $58 \%$ of women aged 18 and over met the guidelines for aerobic activity. According to Public Health England (2017), 41\% of adults aged 40-60 years in England walk less than 10 min continuously each month at a brisk pace and $19.7 \%$ of adults aged $40-60$ years classed as inactive [5].

Although there are no defined guidelines on what should be considered the maximum amount of sedentary behaviour, the one sedentary behaviour considered a health threat is sitting [10]. From the epidemiological evidence, it is becoming clear that the associations of sitting time with all-cause mortality (ACM) and cardiovascular disease (CVD) mortality are often seen to be dependent on moderate to vigorous physical activity levels [11].

This survey aims to report whether there were changes in physical activity, sitting time and eating habits in the UK during the first lockdown of the COVID-19 outbreak as well as reporting perceived barriers to undertaking physical activity. Changes in lifestyle behaviours during the lockdown period may assist in predicting the impact of flexible and home working and whether this might be a lifestyle change that could improve the health of the population in future years and may determine in relation to physical activity, sitting time and physical inactivity. Arguably, more time for physical activity may be available to an individual working from home, but more time may also be spent sitting; therefore, detriments to health will also be considered.

Subsequently, this research sets out to determine whether working from home impacts lifestyle, in particular frequency of physical activity, amount of physical inactivity and sitting time, along with eating habits. This may help to shape future working patterns in the UK.

\section{Methods}

\subsection{Survey Details}

This study was a descriptive analysis of the effect of lockdown during the SARS-CoV-2 pandemic in the UK on lifestyle. A 48-item online survey was created using Qualtrics (Qualtrics ${ }^{\mathrm{XM}}$, Provo, UT, USA) following the NHS Live Well Survey [12], and the International Physical Activity Questionnaire (IPAQ) [13] and adapted with additional questions by the investigators). Some questions were displayed based on skip logic, so many participants responded to fewer than 48 questions. The average time taken to complete the survey was $51 \mathrm{~min}$, with a range from $5 \mathrm{~min}$ to 5 days (after 5 days the questionnaire automatically timed out). When removing the outliers (deemed as those who did not complete the survey, 
taken in less than $8 \mathrm{~min}$ and those who left the survey open and returned some hours later, taken more than $60 \mathrm{~min}$ ) the average time taken to complete the survey was $16 \mathrm{~min}$.

The survey was distributed via email correspondence through students and staff at the University of Hertfordshire and their associates and advertised on social media for a convenience sample of adults aged 18 years and above. Although this sampling may generate a sample that is skewed towards university staff and students, these participants represent those to whom staying at home during a lockdown will have the greatest impact.

Ethical approval was obtained through the University of Hertfordshire's Health, Science, Engineering and Technology Ethics Committee with Delegated Authority, (protocol number LMS/SF/UH/04142) and no identifying data was collected. Consent was given by participation and completing the survey. The survey was live from 29 April to 13 May 2020 which was week five to seven of the UK lockdown, these were the weeks during the stay-at-home phase (phase 1) of the first UK lockdown. During this time people were told to stay inside their homes with limited exceptions for shopping or once a day for exercise such as running walking or cycling and for medical needs including providing care for another or to go to work. Gym and group exercise classes were not allowed during this time. The survey was retired at the 'stay alert stage' which included the phased re-opening of schools, non-essential retail, and more social and family contact.

The questionnaire evaluated the self-reported amount and type of physical activity, sitting time, perceived barriers to undertaking physical activity with regards to tiredness and time, weight change and eating habits for before and during lockdown.

\subsection{Scales and Description of Frequencies}

The frequency of participation in physical activity was ordered such that never $=$ not at all, rarely = once a month, sometimes $=1-2$ times a month, often $=1-2$ times a week and frequently = greater than twice a week.

Physical inactivity is defined as participating in less than $30 \mathrm{~min}$ of moderate intensity physical activity per week as defined by Public Heath England (2017) [14].

\subsection{Data Analysis}

Data were analysed using SPSS (version 26, IBM, New York, NY, USA), Excel (Microsoft Office 365, Microsoft, Irvine, CA, USA) and Social Science Statistics and Descriptive statistics (https: / / www.socscistatistics.com) (accessed on 26 September 2020). A contingency table analysis using Pearson's-Chi squared or Fisher's exact test, where appropriate, were used to determine whether a statistically significant difference was observed pre to during lockdown. All statistical tests were considered statistically significant at $p \leq 0.05$.

\section{Results}

3.1. Descriptive Statistics

\subsubsection{Participants}

A total of 818 participants completed the study (Table 1). 
Table 1. Participant Characteristics $(n=818)$.

\begin{tabular}{|c|c|c|c|c|c|c|c|c|}
\hline Personal Details & $\begin{array}{c}\text { Age (Years) } \\
\text { (Mean } \pm \text { SD) } \\
47 \pm 13\end{array}$ & $\begin{array}{c}\left.\text { BMI (kg.m } \mathbf{~}^{-2}\right) \\
\text { (Mean } \pm \text { SD) } \\
26 \pm 5\end{array}$ & $\begin{array}{c}\text { Males } \\
\text { (Total Number) } \\
177\end{array}$ & $\begin{array}{c}\text { Females } \\
\text { (Total Number) } \\
641\end{array}$ & & & & \\
\hline Education & No Schooling & $\begin{array}{l}\text { GCSE or } \\
\text { equivalent }\end{array}$ & $\begin{array}{c}\text { A level or BTEC or } \\
\text { Apprenticeship or } \\
\text { equivalent }\end{array}$ & Bachelor's degree & Master's degree & Doctorate & & \\
\hline Total number & 3 & 82 & 203 & 279 & 160 & 91 & & \\
\hline Employment & Key Workers & Furloughed & $\begin{array}{l}\text { Working from } \\
\text { home }\end{array}$ & $\begin{array}{l}\text { Working outside } \\
\text { from home }\end{array}$ & Retired & Unemployed & $\begin{array}{c}\text { Carer for family } \\
\text { member }\end{array}$ & \\
\hline Total number & 188 & 113 & 483 & 98 & 37 & 83 & 4 & \\
\hline Income & $<£ 5200$ & $£ 5200$ to $£ 15,999$ & $£ 15,600$ to $£ 25,999$ & $£ 26,000$ to $£ 36,399$ & $£ 36,400$ to $£ 51,999$ & $£ 52,000$ to $£ 77,999$ & $£ 78,000$ and above & Prefer not to say \\
\hline Total number & 3 & 28 & 49 & 83 & 147 & 192 & 217 & 99 \\
\hline Ethnicity & White & Asian & Black & Chinese & Mixed & $\begin{array}{c}\text { Other/Prefer not } \\
\text { to say }\end{array}$ & & \\
\hline Total number & 754 & 26 & 6 & 3 & 12 & 10 & & \\
\hline
\end{tabular}




\subsubsection{Self-Reported Body Mass and Eating Habits}

After 4 weeks of lockdown, self-reported body mass change showed an increase in weight in $32 \%$ of the cohort, with the majority (51\%) staying the same and $17 \%$ reporting a decrease. Changes in food intake showed that $39.1 \%$ of the participants were eating more during the lockdown period compared to before, $15.3 \%$ were eating less and $45.6 \%$ were eating about the same. Participants also reported an increase in the number of portions of 'bad' or unhealthy snacks, high in fat, sugars or salt being eaten daily. An intake of greater than five portions per week increased from 269 to 384 participants, a 30\% increase.

\subsubsection{Changes in Exercise Frequency during Lockdown for Gym and Exercise Class Participation}

Of the 818 participants included in this study, 185 (22.6\%) never went to a gym or undertook an exercise class (including on-line/virtual classes) either before or during lockdown. Of the 242 participants who went to a gym or undertook an exercise class frequently (>2 times a week) before lockdown (Figure 1), 120 (49.6\%) continued this during lockdown, whilst gyms and group exercise classes were closed but home workouts and online classes continued. Sixty-four participants $(26.4 \%)$ of those who frequently ( $>2$ times per week) attended the gym or an exercise class before lockdown changed to never attending the gym or an exercise class during lockdown. Fifteen of the 242 participants $(6.2 \%)$ who had never gone to the gym or undertaken an exercise class changed to frequently participating.

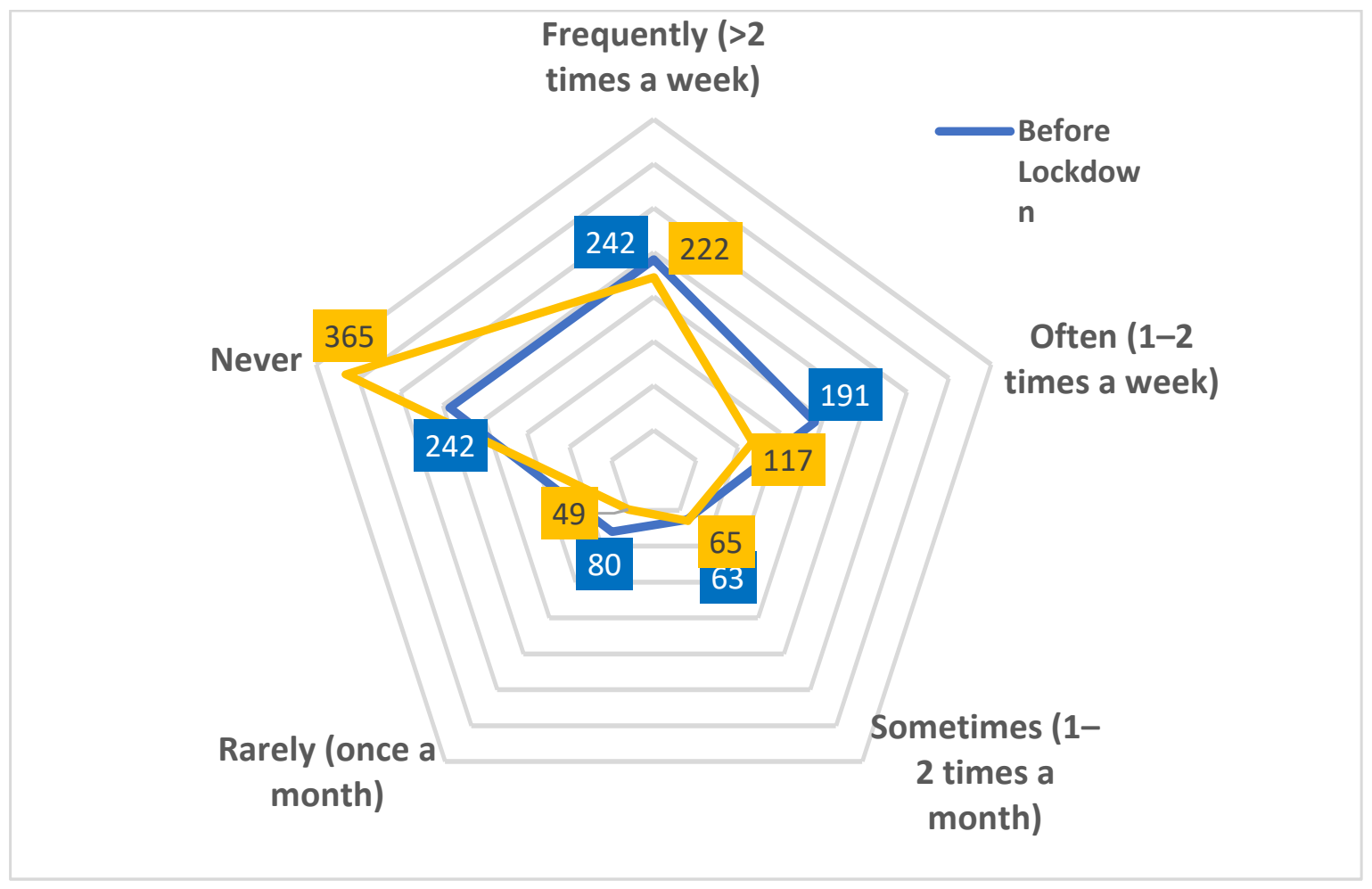

Figure 1. Frequency of using gym or exercise classes before and during lockdown, shown by number of participants for each category.

From pre-lockdown to during lockdown, the total number of participants who never went to the gym or did exercise classes increased from 242 to $365(50.8 \%)$. Those who went to the gym or took classes sometimes, often, or frequently before lockdown reduced from 496 to 404 during lockdown, (18.6\% decrease). The relationship between these variables was significant, $X^{2}(1, N=818) 33.7, p<0.001$.

This data shows a large proportion of the sample that never went to a gym or undertook an exercise class before lockdown continued this behaviour during lockdown. 
Changes in exercise patterns during lockdown showed half of those frequently exercising before lockdown continued to do so; however, a notable proportion of this sub-sample moved from frequent use of gyms and exercise classes to never exercising (26.5\%). Far less participants increased their participation in gym and exercise classes from before lockdown to during.

\subsection{Exercise Modes}

Changes in Outdoor Running and Cycling

From a total of 790 ( 28 non-responders) participants, 300 participants never cycled or ran before or during lockdown, 420 undertook outdoor running or cycling at least once a month before lockdown increasing to 490 during lockdown, a 17\% increase. Moreover, there was a $38 \%$ increase in the number of participants (147 to 203) reporting to exercise more than twice a week (frequently), and a reduction in those who exercised less than once a month from before to during lockdown (559 to 478). This suggests a large change to exercise patterns during lockdown, with more people exercising at least once per month. A chi-square test of independence was performed to examine the relationship between those who exercised frequently before and during lockdown compared to those who exercise sometimes or less. The relationship between these variables was significant, $X^{2}(1, N=790)=14.84, p=0.00012$.

\subsection{Walking Behaviour}

In response to how many times per week participants walked for a minimum of $30 \mathrm{~min}$ at a time, 26 participants never undertook a walk lasting $\geq 30$ min either before or during lockdown. The number who undertook a walk of $\geq 30 \mathrm{~min}$ three or more times a week increased by $70 \%$ from 289 to 492 participants from before to during lockdown. Of the 289 participants who exercised $\geq 30$ min two or more times a week before lockdown 32 $(11 \%)$ reduced to less than once a week during lockdown.

The number of participants walking 150 min per week increased during lockdown resulting in an additional $11.4 \%$ of participants meeting the UK Government guidelines, [4] for moderate-intensity activity (Table 2 ) but with the majority (76\%) still not achieving this through walking. However, with regards to 10 -min daily walks there was a $23 \%$ increase in participants walking $10 \mathrm{~min}$ or more, seven days a week during lockdown compared to before (Table 2).

Table 2. Total number of participants and percentage change from before to during lockdown of those completing $<150$ or $\geq 150$ min of moderate-intensity activity per week and walks lasting $\geq 10 \mathrm{~min}$, seven days a week.

\begin{tabular}{cccc}
\hline Walking Time & Before Lockdown & during Lockdown & $\begin{array}{c}\text { Before to during } \% \\
\text { Change }\end{array}$ \\
\hline$<\mathbf{1 5 0}$ min per week & 639 & 621 & -2.8 \\
$\mathbf{1 0 0}$ min per week & 176 & 194 & 11.4 \\
$\mathbf{1 0}$-min Daily Walks & 244 & 300 & 23 \\
\hline
\end{tabular}

\subsection{Inactivity}

Assuming that no other exercise was taking place 8.6\% (69 participants from 801) would be classified as sedentary from this data. For those classified as physically inactive (less than 30 min walking per week, 307 participants were classified as inactive before lockdown reducing to 267 during lockdown (13\% reduction).

\subsection{Sitting Time}

Increases in percentage change from before to during lockdown for sitting time of $\geq 8 \mathrm{~h}$ per day could be seen on both weekdays (44\%) and weekends (122\%) (Figure 2). Those sitting for $\leq 4 \mathrm{~h}$ per day, percentage change from before to during lockdown reduced 
during weekends $(-25 \%)$ and weekdays $(-47 \%)$, indicating a large increase in those sitting for $\geq 8 \mathrm{~h}$ per day and a noticeable shift in sitting patterns during weekends (Figure 2).

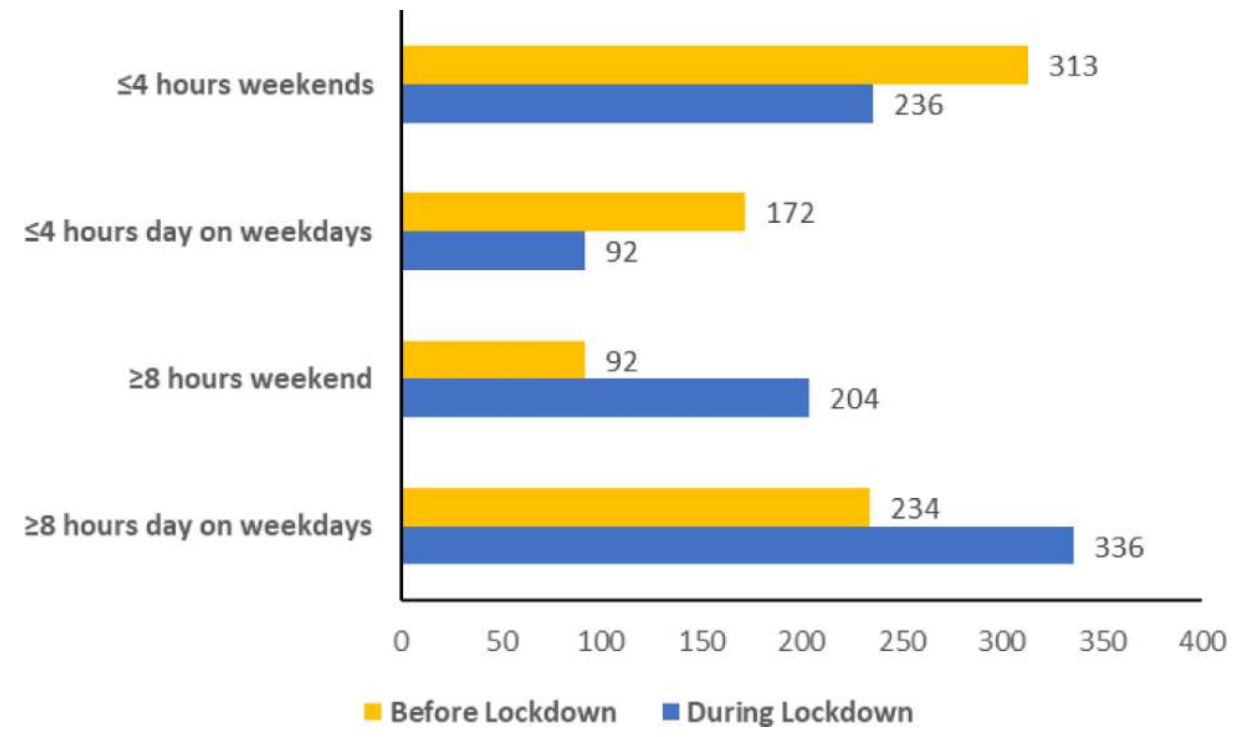

Figure 2. Number of participants sitting for $\leq 4$ and $\geq 8 \mathrm{~h}$ per day on weekdays and weekends before and during lockdown.

\subsection{Barriers to Physical Activity}

Time was a barrier to exercise for 640 participants before lockdown who described not having enough time to exercise either sometimes, often, or most of the time. However, during lockdown this reduced by $60 \%$ to 240 participants. The relationship between these variables was significant, $X^{2}(1, N=818) 367.5, p<0.00001$, showing that participants who stated lack of time to take part in physical activity had significantly decreased during lockdown.

Before lockdown, 578 participants reported not feeling like undertaking physical activity due to tiredness either sometimes, often, or most of the time. During lockdown this decreased by $16 \%$ to 487 participants. These changes in barriers to physical activity concur with the increases shown in walking, running, and cycling activity.

\section{Discussion}

This study surveyed a cohort of the UK adult population to determine changes in physical activity and eating patterns during the first national lockdown against prelockdown measures. The study aimed to determine whether working from home and flexible working impacts lifestyle, in particular frequency and amount of physical activity, sitting time and food intake. The aims of the study were met with the following results.

\subsection{Changes in Physical Activity during Lockdown}

\subsubsection{Frequency of Gym and Exercise Class Participation}

From Figure 1 it can be seen that there was an increase of $50.8 \%$ pre to during lockdown for those who never exercised at the gym or undertook an exercise class (either online or live) and a decrease of $53.3 \%$ during lockdown for those attending $>2$ times per week before lockdown to never during lockdown showing that many frequent exercisers did not engage with online and home workouts. These decreases in exercise suggests that those not meeting the minimum physical activity guidelines set out by the World Health Organisation [6] increased during lockdown. This was partly due to the gyms and health clubs being closed but the option of home and online workouts were freely available, although uptake appears low and does not appear to have positively impacted time spent exercising at home. Only $1.83 \%$ of the participants changed from never going to 
the gym or undertaking an exercise class before lockdown to frequently ( $>2$ times per week) undertaking a gym or exercise class activity (at-home or on-line) during lockdown, suggesting that uptake of online workouts was not widespread.

The ramifications of such a finding are significant given that the risk of cancer, diabetes, osteoporosis, cardiovascular disease and poor mental health amongst individuals that never exercise are markedly elevated [15]. Furthermore, the risk of mortality and experiencing severe symptoms from COVID-19 itself is also likely increased, particularly in individuals with low physical activity levels [16].

\subsubsection{Moderate Exercise and Walking}

This study found that there was a 70\% increase in those walking at least $30 \mathrm{~min}$ continuously 3 or more times a week during lockdown, which although slightly below the minimum recommendation of $150 \mathrm{~min}$ per week is still a positive increase in the amount of PA undertaken by the participants and reduced sedentary behaviours as recommended by the WHO [6]. Current WHO guidelines on PA acknowledge that even a small amount of physical activity is better than none [6].

Of the 73 participants who never walked before lockdown, 26 continued to never walk during lockdown, however 21 of this cohort did start to walk frequently ( $>2$ times per week) during lockdown. The curvilinear dose-response relationship between physical activity and health benefits suggests the greatest proportional benefits occur when progressing from inactive or very low levels of physical activity to moderate levels, even if they are below the minimum threshold recommended by guidelines [4] as seen above.

The increase in physical activity in the form of walking concurs with the findings of a study in Italy [3]. In a subsample of the Italian population physical activity frequency was significantly increased during lockdown compared to pre-lockdown, although there was no change in frequency in those that were not exercising before lockdown. Our results also align with data from an international online survey from four different continents which reported a significant reduction in total time spent doing physical activity, during COVID-19 lockdown compared to before lockdown [17].

In the current study, less than $25 \%$ of participants met the minimum guidelines of $150 \mathrm{~min}$ of moderate intensity physical activity per week either before or during lockdown. In comparison, the UK national estimate of people meeting the minimum guideline of 150 min of moderate intensity exercise as of November 2019 was $67 \%$ for adults aged 19 and over [18]. It should be noted that our sample over-represents females who tend to have lower physical activity levels compared to males in the UK [18], which may explain some of the discrepancy between our sample and national estimates. Furthermore, only walking was used as an estimate of moderate exercise as all other forms of moderate exercise (such as doubles tennis and aqua aerobics) were not available during the lockdown.

National guidelines, recommend including walking, 5 days a week (or 150 min over a week) or $10 \mathrm{~min}$ per day for 7 days per week [5]. The frequency of undertaking a minimum 10 min walk 7 days per week increased from $30 \%$ before lockdown to $37 \%$ during lockdown (Table 2), indicating more participants were able to be physically active each day.

\subsubsection{Outdoor Running and Cycling}

Outdoor exercise patterns changed during lockdown compared to pre-lockdown, as the number of participants exercising by running or cycling increased, with individuals exercising $>2$ times per week showing a $38 \%$ increase. Those exercising less than once a month reduced from 559 to 478 participants, resulting in an increase in those exercising once a month or more. These changes were most likely brought about by the enforced rules that people could only go outside to exercise once a day therefore participants who wished to continue exercising decided to optimise this opportunity by running and cycling. Conversely, those who had no interest in running or cycling before lockdown continued to have no interest during with the majority of participants (300) never cycling 
or running either before or during lockdown. This is not to say that these participants did not undertake more walking as discussed previously.

\subsection{Physical Inactivity}

Physical inactivity, defined as $<30 \mathrm{~min}$ moderate intensity activity per week in the UK, is a risk factor for developing disease and can hinder the ability to resist viral infection [16]. The rate of physical inactivity during lockdown likely increased across the world, with global average step count decreasing up to $38 \%$ on the same period last year according to data from the 30 million global users of wearable technology company FitBit [19]. Our data showed a 13\% decline in individuals performing $<30$ min exercise per week during lockdown compared to pre-lockdown amongst those that were classed as physically inactive before lockdown. Of those that were physically active ( $>30 \mathrm{~min}$ per week) before lockdown, $36 \%$ became physically inactive during lockdown and of the 289 participants who exercise $>30$ min 2 or more times a week before lockdown, $32(11 \%)$ reduced to less than once a week during lockdown. This is a surprising finding of the study where lockdown had caused people to work from home and were able to self-regulate the amount of time spent walking. However, as mentioned above the overall data showed an increase of $70 \%$ for those who walked $>30$ min per week, during lockdown, which would give health benefits to those individuals. However, despite time spent walking $30 \mathrm{~min}$ per week increasing, there was also an increase in the duration of sitting time in our sample during lockdown compared to pre-lockdown.

During weekdays where people are often sedentary due to office work the number of people sitting for $8 \mathrm{~h}$ or more a day rose by $43.6 \%$ from 234 to 336 participants. However, at weekends the number of people sitting for more than $8 \mathrm{~h}$ a day increased by $121 \%$ from 92 people to 204 people. To compound the issue of people being sedentary during lockdown, it was also seen that those sitting for less than $4 \mathrm{~h}$ per day decreased during lockdown during the week and at weekends by $46.5 \%$ and $25.6 \%$, respectively. Similarly, in a Belgian population, sitting time increased during COVID-19 lockdown [20]. The importance of this requires highlighting since increased sitting time ( $>4 \mathrm{~h}$ per day) has been shown to be a risk factor for all-cause mortality, independent of physical activity levels [21] The estimated national average for sitting time in the UK is $9 \mathrm{~h}$ per day, with premature mortality risk increasing for those sitting $>7 \mathrm{~h}$ per day and an additional $5 \%$ greater risk for every hour thereafter [22]. In this study $41.1 \%$ of total participants sat for more than $8 \mathrm{~h} \mathrm{a}$ day on weekdays. Prolonged sitting increases health risk even in those meeting minimum physical activity guidelines [4]. Breaking up prolonged periods of sitting time, even with standing or light activity, has been associated with improved cardio-metabolic health [23]. Evidence suggests 2-3 min of light activity every 20-30 min over several hours improves cardio-metabolic health [24], and would be recommended generally but especially during periods of confinement where sitting time is likely to be increased. Increases in $10 \mathrm{~min}$ walks on 7 days a week seen in this study and discussed above would help to counteract this sedentary behaviour.

\subsection{Body Mass and Eating Habit Changes}

Thirty-two percent of the participants in this study self-reported an increase in body mass after the first 4 weeks of lockdown. This gain in weight may be attributed to changes in eating habits but also changes in physical activity levels and sitting time. The majority of participants (51\%) reported no change in weight with $17 \%$ reporting a decrease. These figures correspond to the changes to eating habits, with $39.1 \%$ of the participants selfreporting an increase in food intake and only $15.3 \%$ eating less. Further to this there was a $30 \%$ increase in those eating five or more portions of 'bad' or unhealthy food each week. An increase in body mass would be expected with this increase in food intake and unhealthy food, despite the increased walking times. Walking does not tend to utilise high amounts of calories and is negated by the increase in reported sitting time and concomitant decrease in exercise frequency, therefore an increase in body mass would be expected. 


\subsection{Barriers to Physical Activity}

Lack of time has been suggested as a barrier to physical activity and this is further supported by our data showing individuals cited having more time to exercise increasing by $60 \%$ during lockdown amongst those who said they felt like not exercising 'sometimes', 'often' or 'most of the time' before lockdown. Another encouraging aspect of the work from home directive during lockdown, was that there was a $16 \%$ decrease in those feeling too tired to exercise. Feeling less tired, along with extra time to exercise, whilst working from home, may help more of the population to achieve recommended physical activity guidelines [4]. It is an aspect of the lockdown which needs to be considered for future working patterns when policy on returning to work is being considered.

This has important implications for promoting better health since the UK Chief Medical Officer recommends daily physical activity [4], suggesting employees and policymakers should reconsider working patterns to enable more time for physical activity to be performed daily.

\subsection{Considerations to Working from Home and Future Lockdowns}

This study shows that increases in frequency of daily walks and amount of weekly walking times and decreases in barriers to exercise such as time and tiredness occurred during the lockdown. We recommend that these results should be considered and recognised in future decision making for people to work from home and have flexible working patterns as they show that people have more time and feel less tired to undertake PA.

Although, other sedentary behaviour and changes in eating patterns may have been negatively impacted by the lockdown, it must be considered that during this time the people of the UK did not have access to sporting or gym facilities and were having to stay indoors, which would not be the case outside of a lockdown.

We recommend practical advice for individuals in case of future lockdowns leading to confinement and limited social interaction, to follow recommendations for physical activity with respect to any guidelines set out by respective authorities such as maintaining social distancing. Individuals should undertake physical activity as frequently as possible, preferably daily, to maintain good health during any lockdown periods [16] and in everyday life. Utilisation of technology such as online classes, wearable sensors and mobile applications can provide social interaction and motivation to become more physically active in a socially distanced environment. However, when society opens up fully again, the additional benefits of time and lack of tiredness, as seen during this lockdown may assist those working from home to undertake more PA.

\subsection{Strengths/Limitations and Future Work}

Results from our study must be interpreted with respect to limitations. Individuals were required to self-report thus potentially introducing some bias into the findings. Our dataset also over-represented females and the white ethnicity which may have influenced responses to certain questions. Online surveys are not always representative of the wider population and our sample size was relatively small for an online survey and as advertised through the University, as well as on social media, may have attracted more students than the general population, full descriptive and demographic information can be found in Table 1. However, given restrictions on social interaction due to lockdowns, online surveys were utilised similar to other studies during the COVID-19 pandemic $[3,17]$. A strength of our study was determining responses during the peak of the COVID-19 lockdown, however future work should consider determining the long-term changes to lifestyle patterns following the COVID-19 lockdown and whether patterns are influenced by future lockdowns. Further baseline data was self-reported along with week 5 data, which was a limitation of data collection during the COVID-19 lockdowns.

Future work may also consider sub-group analysis based on independent variables (age, sex, ethnicity, household income, physical activity levels) on lifestyle patterns to minimise the imbalances in sub-samples of the population as well as looking at the impact 
on family life and time spent exercising with children. Lastly, a qualitative analysis of reasons as to why lifestyle patterns were or were not changed during lockdown would be useful to inform public health and policy.

\section{Conclusions}

During lockdown time spent walking, running and cycling increased; however, the majority of people still did not meet the Government guidelines for physical activity. Less people cited lack of time and feelings of tiredness as barriers to PA during lockdown, which should be considered in future flexible working and working from home policies. Sitting time increased both during weekdays and weekend days along with food intake during lockdown. Gains are especially significant for those currently doing the lowest levels of activity (fewer than 30 min per week), as the improvements in health per additional minutes of physical activity will be proportionately greater, and this was the area that most improvements were found.

Regular PA and breaking up prolonged periods of sitting time are important in the prevention of severe health complications during lockdowns and during everyday life and to meet the Government and WHO guidelines for PA. However, more time and less tiredness may encourage people to undertake more PA when working from home.

Author Contributions: Conceptualization L.K. and A.W., methodology L.K., A.W. and D.M., software L.K., A.W. and T.D., validation L.K. and A.W., formal analysis L.K. and K.S., investigation L.K. and A.W., resources L.K., data curation L.K., K.S., T.D. and A.W., writing-original draft preparation L.K. and T.D., writing - review and editing D.M. and A.W., visualization L.K. and A.W. All authors have read and agreed to the published version of the manuscript.

Funding: This research received no external funding.

Institutional Review Board Statement: The study was conducted according to the guidelines of the Declaration of Helsinki, and approved by the University of Hertfordshire's Health, Science, Engineering and Technology Ethics Committee with Delegated Authority, (protocol number LMS/SF/UH/04142 approved 24 April 2020).

Informed Consent Statement: Informed consent was obtained by all subjects involved in the study.

Data Availability Statement: Data underpinning this publication are available at https://doi.org/ $10.18745 /$ ds. 25047 .

Conflicts of Interest: The authors declare no conflict of interest.

\section{References}

1. World Health Organisation. Coronavirus Disease (COVID-19) Pandemic. 2020. Available online: https://www.euro.who. int/en/health-topics/health-emergencies/international-health-regulations/news/news/2020/2/2019-ncov-outbreak-is-anemergency-of-international-concern (accessed on 21 February 2021).

2. GOVUK. UK Government Prime Minister's Statement on Coronavirus (COVID-19): 23 March 2020. 2020. Available online: https: / / www.gov.uk/government/speeches/pm-address-to-the-nation-on-coronavirus-23-march-2020 (accessed on 10 July 2020).

3. Di Renzo, L.; Gualtieri, P.; Pivari, F.; Soldati, L.; Attinà, A.; Cinelli, G.; Cinelli, G.; Leggeri, C.; Caparello, G.; Barrea, L.; et al. Eating habits and lifestyle changes during COVID-19 lockdown: An Italian survey. J. Transl. Med. 2020, 18, 1-15. [CrossRef] [PubMed]

4. Davies, D.S.C.; Atherton, F.; McBride, M.; Calderwood, C. UK Chief Medical Officers' Physical Activity Guidelines; Department of Health and Social Care, Chief Medical Officer: London, UK, 2019; pp. 1-65.

5. Public Health England. 10 Minutes Brisk Walking Each Day in Mid-Life for Health Benefits and towards Achieving Physical Activity Recommendations: Evidence Summary. 2017. Available online: https://assets.publishing.service.gov.uk/government/ uploads/system/uploads /attachment_data/file/639030/Health_benefits_of_10_mins_brisk_walking_evidence_summary.pdf (accessed on 3 February 2021).

6. Bull, F.C.; Al-Ansari, S.S.; Biddle, S.; Borodulin, K.; Buman, M.P.; Cardon, G.; Carty, C.; Chaput, J.P.; Chastin, S.; Chou, R.; et al. World Health Organization 2020 guidelines on physical activity and sedentary behaviour. Br. J. Sport. Med. 2020, 54, 1451-1462. [CrossRef] [PubMed]

7. British Heart Foundation [Internet]. Physical Inactivity and Sedentary Behaviour Report. British Heart Foundation. 2017. Available online: https://www.bhf.org.uk/informationsupport/publications/statistics/physical-inactivity-report-2017 (accessed on 3 February 2021). 
8. Public Heath England. Everybody Active, Every Day; Public Heath England: London, UK, 2014.

9. Scholes, S. Health Survey for England 2016 Physical Activity in Adults. 2017. Available online: http://healthsurvey.hscic.gov.uk/ media/63730/HSE16-Adult-phy-act.pdf (accessed on 5 March 2021).

10. Stamatakis, E.; Ekelund, U.; Ding, D.; Hamer, M.; Bauman, A.E.; Lee, I.-M. Is the time right for quantitative public health guidelines on sitting? A narrative review of sedentary behaviour research paradigms and findings. Br. J. Sport. Med. 2019, 53, 377-382. [CrossRef] [PubMed]

11. Petersen, C.B.; Bauman, A.; Grønbæk, M.; Helge, J.W.; Thygesen, L.C.; Tolstrup, J.S. Total sitting time and risk of myocardial infarction, coronary heart disease and all-cause mortality in a prospective cohort of Danish adults. Int. J. Behav. Nutr. Phys. Act. 2014, 11, 13. [CrossRef] [PubMed]

12. NHS. NHS Live Well. 2019. Available online: https://www.nhs.uk/live-well/sleep-and-tiredness/how-to-get-to-sleep/ (accessed on 22 January 2021).

13. Hagströmer, M.; Oja, P.; Sjöström, M. The International Physical Activity Questionnaire (IPAQ): A study of concurrent and construct validity. Public Health Nutr. 2006, 9, 755-762. [CrossRef]

14. Public Heath England. BRISK Walking and Physical Inactivity in 40 to 60 Year Olds. 2017. Available online: https:// documentcloud.adobe.com/link/review? uri=urn:aaid:scds:US:d504adec-351a-4d9d-8e50-2f4668601c0c (accessed on 5 March 2021).

15. World Health Organisation. Physical Inactivity a Leading Cause of Disease and Disability, Warns WHO. 2002. Available online: https:/ / www.who.int/news/item/04-04-2002-physical-inactivity-a-leading-cause-of-disease-and-disability-warns-who (accessed on 5 March 2021).

16. Woods, J.A.; Hutchinson, N.T.; Powers, S.K.; Roberts, W.O.; Gomez-Cabrera, M.C.; Radak, Z.; Berkes, I.; Boros, A.; Boldogh, I.; Leeuwenburgh, C.; et al. The COVID-19 pandemic and physical activity. Sport. Med. Health Sci. 2020, 2, 55-64. [CrossRef] [PubMed]

17. Ammar, A.; Brach, M.; Trabelsi, K.; Chtourou, H.; Boukhris, O.; Masmoudi, L.; Bouaziz, B.; Bentlage, E.; How, D.; Ahmed, M.; et al. Effects of COVID-19 Home Confinement on Eating Behaviour and Physical Activity: Re1sults of the ECLB-COVID19 International Online Survey. Nutrients 2020, 12, 1583. [CrossRef] [PubMed]

18. NHS. Statistics on Obesity, Physical Activity and Diet, England, 2020. 2020. Available online: https://digital.nhs.uk/data-andinformation/publications/statistical/statistics-on-obesity-physical-activity-and-diet/england-2020 (accessed on 25 February 2021).

19. FitBit. FITBIT News: The Impact of Coronavirus on Global Activity. 2020. Available online: https://blog.fitbit.com/covid-19 -global-activity / (accessed on 6 April 2021).

20. Constandt, B.; Thibaut, E.; De Bosscher, V.; Scheerder, J.; Ricour, M.; Willem, A. Exercising in Times of Lockdown: An Analysis of the Impact of COVID-19 on Levels and Patterns of Exercise among Adults in Belgium. Int. J. Environ. Res. Public Health 2020, 17, 4144. [CrossRef]

21. Van Der Ploeg, H.P.; Chey, T.; Korda, R.J.; Banks, E.; Bauman, A. Sitting time and all-cause mortality risk in 222497 Australian adults. Arch. Intern. Med. 2012, 172, 494-500. [CrossRef] [PubMed]

22. Buckley, J.P.; Hedge, A.; Yates, T.; Copeland, R.J.; Loosemore, M.; Hamer, M.; Bradley, G.; Dunstan, D.W. The sedentary office: A growing case for change towards better health and productivity. Expert statement commissioned by Public Health England and the Active Working Community Interest Company. Br. J. Sport. Med. 2015, 49, 1357-1362. [CrossRef] [PubMed]

23. Benatti, A.; Ried-Larsen, M. The Effects of Breaking up Prolonged Sitting Time: A Review of Experimental Studies. Med. Sci. Sports Exerc. 2015, 47, 2053-2061. [CrossRef] [PubMed]

24. Chastin, S.F.M.; Egerton, T.; Leask, C.; Stamatakis, E. Meta-analysis of the relationship between breaks in sedentary behavior and cardiometabolic health. Obesity 2015, 23, 1800-1810. [CrossRef] [PubMed] 